\title{
DARBOUX PROBLEM WITH A DISCONTINUOUS RIGHT-HAND SIDE
}

\section{P. PIKUTA}

Received May 30, 2003 and, in revised form, May 13, 2005

\begin{abstract}
We prove an existence theorem for the Darboux Problem $u_{x y}(x, y)=g(u(x, y)), u(x, 0)=u(0, y)=0$, where $g$ is bounded and measurable.
\end{abstract}

\section{Introduction}

In this paper we consider the Darboux Problem

$$
\begin{aligned}
\frac{\partial^{2} u}{\partial x \partial y}(x, y) & =g(u(x, y)) \quad \text { a.e. in }[0,1] \times[0,1], \\
u(x, 0) & =u(0, y)=0,
\end{aligned}
$$

where $g: \mathbb{R} \rightarrow[a, b], 0<a<b<+\infty$ is supposed to be Lebesgue measurable. The problem arises as a natural extension of the Cauchy Problem for an autonomous equation $x^{\prime}(t)=f(x(t))$ with a discontinuous right-hand side, see [1].

When considering Darboux Problem for equations $u_{x y}=f(x, y, u)$ or $u_{x y}=f\left(x, y, u, u_{x}, u_{y}\right)$, most authors assume Carathéodory-type conditions, i.e. $f$ is measurable with respect to the first two variables, continuous

2000 Mathematics Subject Classification. 35R05, 34K99.

Key words and phrases. Discontinuous Darboux Problem, functional differential equations.

ISSN 1425-6908（C) Heldermann Verlag. 
or Lipschitz with respect to the others, and bounded by an integrable function $M(x, y)$, see e.g. [3], [4], [5], [6]. It should also be mentioned that there is a large number of articles on Darboux Problem in Banach spaces, see e.g. [9], where it is assumed that $f$ is continuous and satisfies a condition expressed in terms of measure of noncompactness. Another approach to the Darboux Problem is to consider $u_{x y}$ the mixed approximate derivative, see $[2]$.

Although the main purpose of this paper is to establish an existence theorem for (1)-(2), the method used here, see also see [7] involves functional differential equations, namely, we deal with the problem

$$
\begin{aligned}
& q^{\prime}(t)=g\left(\int_{0}^{t} \frac{q(s)}{s} d s\right) \quad \text { a.e. in } \mathrm{t} \in[0,1] \\
& q(0)=0
\end{aligned}
$$

where $g: \mathbb{R} \rightarrow[a, b], 0<a<b<+\infty$, is Lebesgue measurable.

Throughout this paper the term measure instead of Lebesgue measure $\mu$ is used and also other concepts such as measurability and integrability are understood as Lebesgue measurability and Lebesgue integrability. We denote by $C[0,1]$ the normed linear space of all continuous functions $x:[0,1] \rightarrow \mathbb{R}$ with the norm $\|x\|=\sup _{t \in[0,1]}|x(t)|$.

\section{Functional equation $q^{\prime}(t)=g\left(\int_{0}^{t} \frac{q(s)}{s} d s\right)$}

First we remind two facts which we need further in the proof of Theorem 2.1 .

Lemma 2.1. Assume that $f:[A, B] \rightarrow \mathbb{R},-\infty<A<B<+\infty$ is continuous and has bounded variation in $[A, B]$. Then $f$ is absolutely continuous if and only if $\mu(f(E))=0$ for every $E \subset[A, B]$ such that $\mu(E)=0[8$, Theorem 4, p. 314].

Lemma 2.2. Assume that $f:[A, B] \rightarrow \mathbb{R},-\infty<A<B<+\infty$ is continuous. If $F \subset[A, B]$ is measurable and $\mu(f(E))=0$ for every $E \subset[A, B]$ such that $\mu(E)=0$, then $f(F)$ is measurable [8, Corollary 2, p. 219].

Theorem 2.1. If $g: \mathbb{R} \rightarrow[a, b], 0<a<b<+\infty$ is measurable, then the problem (3)-(4) has a solution. 
Proof. Proof will be divided into several steps.

1. Define

$$
\begin{aligned}
Z=\{x \in C[0,1]: x(0)=0, a(t-\tau) \leq x(t)-x(\tau) \leq b(t-\tau), \\
0 \leq \tau<t \leq 1\} .
\end{aligned}
$$

For each $f, g \in Z$ and $\alpha \in[0,1]$ we have $\alpha f+(1-\alpha) g \in Z$. Thus $Z$ is convex. Moreover $0 \leq f(t) \leq b$ and

$$
\begin{aligned}
& f(t)-f(\tau) \leq b(t-\tau)=b|t-\tau| \\
& f(\tau)-f(t) \leq a(\tau-t) \leq a|t-\tau| \leq b|t-\tau|
\end{aligned}
$$

for all $t, \tau \in[0,1], t>\tau$. Therefore $|f(t)-f(\tau)| \leq b|t-\tau|, t, \tau \in[0,1]$ and $Z$ is compact.

2. We claim that $A: Z \rightarrow Z$, defined by

$$
(A q)(t)=\int_{0}^{t} g\left(\int_{0}^{z} \frac{q(s)}{s} d s\right) d z, \quad t \in[0,1],
$$

is continuous.

2a. For $q \in Z$ define $h:[0,1] \rightarrow \mathbb{R}$,

$$
h(z)=\int_{0}^{z} \frac{q(s)}{s} d s .
$$

The function $h$ is continuous, strictly increasing and for each $t, \tau \in[0,1]$, $t>\tau$, satisfies

$$
a(t-\tau) \leq h(t)-h(\tau)=\int_{\tau}^{t} \frac{q(s)}{s} d s \leq b(t-\tau) .
$$

Thus $h \in Z$ and, for each $u, v \in h([0,1])$, we have

$$
\left|h^{-1}(u)-h^{-1}(v)\right| \leq \frac{1}{a}\left|h\left(h^{-1}(u)\right)-h\left(h^{-1}(v)\right)\right|=\frac{1}{a}|u-v|,
$$

so $h^{-1}$ is absolutely continuous.

Because $h \in Z$ is continuous and strictly monotonic on $[0,1], h^{-1}$ is continuous and strictly monotonic on a closed interval $h([0,1])$. Thus $h^{-1}$ is of bounded variation on $h([0,1])$. By Lemma 2.1 and Lemma 2.2, $(g \circ h)^{-1}(P)=h^{-1}\left(g^{-1}(P)\right)$ is measurable for every open interval $P \subset h([0,1])$. Hence, $g(h(\cdot))$ is measurable and $A q$ is well defined.

Observe that $A q \in Z$, because $(A q)(0)=0$ and for all $\tau, t \in[0,1], t>\tau$,

$$
a(t-\tau) \leq(A q)(t)-(A q)(\tau)=\int_{\tau}^{t} g\left(\int_{0}^{z} \frac{q(s)}{s} d s\right) d z \leq b(t-\tau) .
$$


2b. Take $\varepsilon>0$ and any sequence $q_{n} \in Z, n \in \mathbb{N}$, convergent (uniformly) to $q \in Z$. Define $h_{n}:[0,1] \rightarrow \mathbb{R}$,

$$
h_{n}(z)=\int_{0}^{z} \frac{q_{n}(s)}{s} d s, \quad n \in \mathbb{N} .
$$

By Lusin's theorem there exists a compact set $K \subset[0, b]$ such that $g_{\mid K}: K \rightarrow[a, b]$ is continuous and

$$
\mu([0, b] \backslash K)<\frac{a \varepsilon}{8 b} .
$$

Since $g_{\mid K}$ is uniformly continuous, there exists $\delta>0$ such that $|u-v|<\delta$, $u, v \in K$ implies $|g(u)-g(v)|<\varepsilon / 2$.

For $z \leq \delta /(2 b+1)$ we have

$$
\begin{aligned}
\left|h_{n}(z)-h(z)\right| & \leq \int_{0}^{z} \frac{\left|q_{n}(s)-q(s)\right|}{s} d s \\
& \leq \int_{0}^{\delta /(2 b+1)} \frac{\left|q_{n}(s)-q(s)\right|}{s} d s \\
& \leq \frac{2 b \delta}{2 b+1}<\delta .
\end{aligned}
$$

If $z>\delta /(2 b+1)$, then

$$
\begin{aligned}
\left|h_{n}(z)-h(z)\right| & \leq \int_{0}^{z} \frac{\left|q_{n}(s)-q(s)\right|}{s} d s \\
& =\int_{0}^{\delta /(2 b+1)} \frac{\left|q_{n}(s)-q(s)\right|}{s} d s+\int_{\delta /(2 b+1)}^{z} \frac{\left|q_{n}(s)-q(s)\right|}{s} d s \\
& \leq \frac{2 b \delta}{2 b+1}+\int_{\delta /(2 b+1)}^{z} \frac{\left\|q_{n}-q\right\|}{s} d s \\
& \leq \frac{2 b \delta}{2 b+1}+\left\|q_{n}-q\right\| \int_{\delta /(2 b+1)}^{1} \frac{d s}{s} \\
& \leq \frac{2 b \delta}{2 b+1}+\left\|q_{n}-q\right\| \ln \frac{2 b+1}{\delta} .
\end{aligned}
$$

Since $\left\|q_{n}-q\right\| \rightarrow 0, n \rightarrow \infty$, there exists $n_{0}$ such that

$$
\left\|q_{n}-q\right\|<\frac{\delta}{2 b+1}\left(\ln \frac{2 b+1}{\delta}\right)^{-1}
$$

for $n>n_{0}$. Therefore for $n>n_{0}$ and each $z \in[0,1]$, we have

$$
\left|h_{n}(z)-h(z)\right| \leq \sup _{z \in[0,1]}\left|\int_{0}^{z} \frac{q_{n}(s)}{s} d s-\int_{0}^{z} \frac{q(s)}{s} d s\right|<\delta .
$$


2c. Fix $n>n_{0}$ and define $F=h^{-1}(K) \cap h_{n}^{-1}(K)$. We have

$$
\begin{aligned}
{[0,1] \backslash F } & =[0,1] \backslash\left(h^{-1}(K) \cap h_{n}^{-1}(K)\right) \\
& =\left([0,1] \backslash h^{-1}(K)\right) \cup\left([0,1] \backslash h_{n}^{-1}(K)\right) \\
& =\left(h^{-1}([0, b]) \backslash h^{-1}(K)\right) \cup\left(h_{n}^{-1}([0, b]) \backslash h_{n}^{-1}(K)\right) \\
& =h^{-1}([0, b] \backslash K) \cup h_{n}^{-1}([0, b] \backslash K)
\end{aligned}
$$

and using (5) we get

$$
\begin{aligned}
\mu([0,1] \backslash F) & \leq \mu\left(h^{-1}([0, b] \backslash K)\right)+\mu\left(h_{n}^{-1}([0, b] \backslash K)\right) \\
& =\int_{h^{-1}([0, b] \backslash K)} d z+\int_{h_{n}^{-1}([0, b] \backslash K)} d z \\
& =\int_{[0, b] \backslash K}\left(h^{-1}\right)^{\prime}(u) d u+\int_{[0, b] \backslash K}\left(h_{n}^{-1}\right)^{\prime}(u) d u \\
& \leq \frac{\mu([0, b] \backslash K)}{a}+\frac{\mu([0, b] \backslash K)}{a} \leq \frac{\varepsilon}{4 b} .
\end{aligned}
$$

Applying 2b, we obtain

$$
\begin{aligned}
\left\|A q_{n}-A q\right\|= & \sup _{t \in[0,1]}\left|\int_{0}^{t} g\left(h_{n}(z)\right) d z-\int_{0}^{t} g(h(z)) d z\right| \\
\leq & \sup _{t \in[0,1]} \int_{0}^{t}\left|g\left(h_{n}(z)\right)-g(h(z))\right| d z \\
= & \int_{0}^{1}\left|g\left(h_{n}(z)\right)-g(h(z))\right| d z \\
= & \int_{F}\left|g\left(h_{n}(z)\right)-g(h(z))\right| d z \\
& +\int_{[0,1] \backslash F}\left|g\left(h_{n}(z)\right)-g(h(z))\right| d z \\
\leq & \mu(F) \cdot \frac{\varepsilon}{2}+\frac{\varepsilon}{4 b} \cdot 2 b \leq \frac{\varepsilon}{2}+\frac{\varepsilon}{2}=\varepsilon .
\end{aligned}
$$

Thus $A: Z \rightarrow Z$ is continuous.

3. It follows from Schauder's fixed point theorem that $A$ has a fixed point in $Z$. Thus the problem (3)-(4) has a solution.

\section{Darboux Problem}

Definition 3.1. We say that a continuous function $u$ : $[0,1] \times[0,1] \rightarrow \mathbb{R}$ is a solution to the Darboux Problem (1)-(2) if $u$ satisfies the equation (1) a.e. in $[0,1] \times[0,1]$ and the initial condition $(2)$ for $x, y \in[0,1]$. 
Theorem 3.1. If $g: \mathbb{R} \rightarrow[a, b], 0<a<b<+\infty$, is measurable, then the problem (1)-(2) has a solution.

Proof. Let $q$ be a solution to the problem (3)-(4). Define $v:[0,1] \rightarrow \mathbb{R}$,

$$
v(t)=\int_{0}^{t} \frac{q(s)}{s} d s, \quad t \in[0,1]
$$

and $u:[0,1] \times[0,1] \rightarrow \mathbb{R}$

$$
u(x, y)=v(x y), \quad(x, y) \in[0,1] \times[0,1] .
$$

We have

$$
\frac{\partial^{2} u}{\partial x \partial y}(x, y)=v^{\prime}(x y)+x y \cdot v^{\prime \prime}(x y)=q^{\prime}(x y)=g(u(x, y))
$$

a.e. in $(x, y) \in[0,1] \times[0,1]$. Obviously, $u(x, 0)=u(0, y)=v(0)=0$. Thus $u$ is a solution to the problem (1)-(2).

\title{
References
}

[1] Binding, P., The differential equation $\dot{x}=f \circ x$, J. Differential Equations 31 (1979), 183-199.

[2] Bugajewski, D., Szufla, S., On the Aronszajn property for differential equations and the Denjoy integral, Comment. Math. Prace Mat. 35 (1995), 61-69.

[3] De Blasi, F. S., Myjak, J., On the structure of the set of solutions of the Darboux problem for hyperbolic equations, Proc. Edinburgh Math. Soc. (2) 29(1) (1986), 7-14.

[4] Deimling, K., A Carathéodory theory for systems of integral equations, Ann. Mat. Pura Appl. (4) 86 (1970), 217-260.

[5] Gugielmino, F., Sulle risoluzione del problema di Darboux per l'equazione $s=$ $f(x, y, z)$ (in Italian), Bol. Un. Mat. Ital. (3) 13 (1958), 308-318.

[6] Idczak, D., Nonlinear Goursat-Darboux problem and its optimization, XII ${ }^{\text {th }}$ International Conference on "Nonlinear Oscillations" (Cracow, 1990), Nonlinear Vib. Probl. 25 (1993), 143-157.

[7] Pikuta, P., Rzymowski, W., A discontinuous functional differential equation, J. Math. Anal. Appl. 277 (2003), 122-129.

[8] Rado, T., Reichelderfer, P. V., Continuous Transformations in Analysis, SpringerVerlag, Berlin-Göttingen-Heidelberg, 1955.

[9] Rzepecki, B., On the hyperbolic equation in Banach spaces, Demonstratio Math. 23(3) (1990), 713-718.

\author{
Piotr Pikuta \\ Institute of Mathematics \\ Maria Curie-SkŁodowska University \\ Pl. M. Curie-Sklodowskiej 1 \\ 20-031 Lublin, Poland \\ E-MAIL:PPIKUTA@GOLEM.UMCS.LUBLIN.PL
}

